\title{
Experimental Investigation of the Mechanical Behavior and Permeability of 3D Printed Sandstone Analogues Under Triaxial Conditions
}

\author{
J. S. Gomez ${ }^{1}$ (D) R. J. Chalaturnyk ${ }^{1} \cdot$ G. Zambrano-Narvaez ${ }^{1}$
}

Received: 30 April 2018 / Accepted: 12 October 2018 / Published online: 27 October 2018

(C) The Author(s) 2018

\begin{abstract}
Consolidated drained triaxial tests arise as one of the most exhaustive methods to quantify the strength, volumetric behavior and failure process of rocks. Understanding the compressibility of a rock matrix and the permeability evolution induced by the effects of confining stress is essential to achieve a better understanding of the productive behavior and performance of enhanced hydrocarbon recovery methods in natural reservoirs. This study investigates the suitability of using of reservoir sandstone analogues, 3D printed with silica sand, to analyze the behavior of natural rocks. A comprehensive mechanical and hydraulic characterization of $3 \mathrm{D}$ printed silica sand is addressed by subjecting the sandstone analogues to various levels of confining stress and measuring their cumulative volumetric deformation and permeability evolution at each compression stage. Experimental results demonstrate that $3 \mathrm{D}$ printing technology can reproduce porous media that resembles the mechanical behavior of natural reservoir rocks. Nonetheless, some divergences are encountered with the properties of 3D printed analogues when compared to natural reservoir rocks. The 3D printed sandstone analogues were found to be more compressible and permeable than widely studied reservoirs such as Berea Sandstone. Efforts are made to optimize the 3D printing process of the rock analogues to overcome the differences encountered in the mechanical and hydraulic behavior.
\end{abstract}

Keywords Additive manufacturing technology $\cdot 3 \mathrm{D}$ printing $\cdot$ Sandstone analogues . Compressibility $\cdot$ Permeability

\section{Introduction}

A quick expansion of additive manufacturing technology, referred to as 3D printing, has resulted in the continuous growth of this technique as an alternative solution to various research inquiries in different scientific fields (Lee et al. 2007; Tymrak et al. 2014; Rankin et al. 2014). Furthermore, the reduced costs of 3D printing systems, open-source software, and

$凶 \quad$ J.S. Gomez

jsgomez@ualberta.ca

1 Reservoir Geomechanics Research Group [RG2], Department of Civil and Environmental Engineering, University of Alberta, Edmonton, AB, Canada 
free access to digital model repositories have strengthened this option as a leading research pathway. Among these research fields, geoscience is poised to use 3D printing to bridge the gap between computational and experimental analyses by transferring digital rock models with specific reservoir characteristics to actual tangible specimens (referred as a "proxies", Ishutov et al. 2015), which can be tested under standard laboratory procedures.

Study of 3D printed "reservoir rocks" can serve as valuable proxies in experimental tests of rock strength, deformation and failure modes. These proxies can help minimizing the influence of inter-specimen variability and specimen disturbance within the same experimental frame. Currently, 3D printed sandstones are being used at the University of Alberta for analyzing the influence of pre-existing fissures on the mechanical behavior of rocks and rock discontinuities in direct shear under constant load/stiffness boundary conditions.

Although initial attempts show potential for the use of 3D printing technology in rock deformation and fluid flow experiments (Head and Vanorio 2016), limitations, particularly with respect to material properties (Jiang and Zhao 2015), are well recognized. The inherent properties of materials commonly used in 3D printing (i.e. plastics, metal powders and gypsum powders) do not essentially resemble those of natural reservoir rocks. There is an urgent need to utilize materials that imitate the behavior of rocks under natural reservoir conditions in a more reliable manner.

In addition to the improvements to the mechanical behavior of the sandstone analogues, the use of silica sand as the printing material has permitted the manufacture of specimens with a more rock-like porous structure (Gomez et al. 2017). Moreover, a granulated material such as silica sand allows control of certain statistical structural properties including grain geometry, grain size distribution and porosity, which ultimately results in a significant reduction of specimen-to-specimen heterogeneity and, conversely, exact control over the explicit inclusion of heterogeneities within any 3D printed sandstone specimen (Primkulov et al. 2017). In contrast with natural materials, which are indeed heterogeneous and anisotropic (Marinos and Hoek 2001; Bewick et al. 2009; Menezes and Lempp 2018), 3D printed sandstone analogues enable the possibility of testing specimens with virtually equivalent matrix structures and in the presence of discontinuities and orientation.

While 3D printing is a promising technology for replicating pore geometry and texture of natural rocks (Ishutov et al. 2015; Gomez et al. 2017; Primkulov et al. 2017), it is imperative to investigate the basic mechanical and flow behavior of sandstone analogues under highpressure conditions, and establish their similarity with the phenomena observed in natural rocks. To achieve this objective, several isotropic consolidated drained triaxial tests were conducted on four 3D printed sandstone analogues, while adjusting other major parameters, such as grain size distribution, porosity, content of cementing material, bedding orientation and specimen dimensions. The particle size distribution of the silica sand had D10,D50 and $D 90$ of 110,175 , and $220 \mu \mathrm{m}$, respectively. D50 was the median grain size. Based on the results obtained from each test, compressibility and volumetric behavior were determined, as well as calculations of stress-strain relationships, strength and deformation behavior, and permeability evolution with the increase of hydrostatic confining pressure. The results obtained from this study contribute to the hypothesis that the 3D printing rock analogues can provide a valuable tool to validate numerical models, develop scaling laws and quantify the degree of influence of pore geometry, fracture network characteristics, and structural heterogeneity on rock's macroscopic properties (Ishutov et al. 2015). 


\section{Methodology}

This section contains a detailed description of the procedure followed to manufacture the reservoir sandstone analogues. Unless otherwise stated, the specimens referred in the forthcoming segments are printed using the same manufacturing properties and are subject to the same thermal curing process (term to be defined in Sect. 2.3) to ensure the complete polymerization of the cementing resin within the pore structure of the analogue specimens.

\subsection{D Printing Sandstone Analogues}

The four cylindrical specimens used in this study were $63.5 \mathrm{~mm}$ in diameter ( 2.5 inch.) by $127 \mathrm{~mm}$ in length (5 inch.). They were 3D printed using the Binder Jetting technology of the Ex-One M-FLEX system (Ex-One 2014), located in the GeoPRINT facility (University of Alberta); developed as part of the Energi Simulation Industrial Research Consortia on Reservoir Geomechanics, by the Reservoir Geomechanics Research Group [RG] ${ }^{2}$. The overall printing process involved three core steps: (1) mixing the silica sand with p-toluene sulphonic acid (acidic activator), (2) alternating deposition of activated silica sand and Furfuryl alcohol (FA) binding material, which was selectively deposited on the build platform, and (3) thermal curing of specimens in a furnace at a constant temperature. Primkulov et al. (2017) provided a detailed description of the printing process used for the sandstone analogues.

The sandstone analogues are built using a specific configuration of three main 3D printing parameters (manufacturing properties): (1) binder saturation, (2) layer orientation, and (3) layer thickness. Binder saturation is defined as the percentage in volume of void spaces occupied by acid cross-linked Furfuryl alcohol (poly-Furfuryl alcohol-PFA). There is a direct relationship between PFA saturation and the peak strength of the rock analogues: the higher the amount of PFA, the stronger in compression is the specimen. Printing layer orientation is expressed as the angle between the building planes inclination and the direction of the axial load application in a conventional compression test. This property affects the heterogeneous behavior of both peak strength and failure mode of the specimens during conventional compression tests, similar to natural layered reservoir rocks. Printing layer thickness is the width along the $Z$-axis of an individual building sand plane. There is an indirect relationship between layer thickness and peak strength of sandstone analogues with a fixed binding saturation: the thicker the building layers, the greater the peak strength.

\subsection{Thermal Curing Process}

Primkulov et al. (2017) found that the peak strength of the sandstone analogues could be highly improved by subjecting the specimens to a high-temperature curing period immediately after being printed. The physics behind the mechanism of a specimen's curing process involved the exothermic reaction generated between the acidic activator and FA, which occurred immediately when they were in contact with each other. This chemical process generated a certain amount of heat that helped the FA to polymerize inside the pore structure of the sandstone analogues.

Nevertheless, the amount of PFA that is cured depends on the equilibrium between the reaction's energy and the FA evaporation rate to the atmosphere. In addition, at the last stages of curing, one of the products of this reaction when it is completed at the atmospheric temperature, is water. Once generated, this fluid is going to remain inside the specimen's pores and can generate ring hydrolysis of the monomers (McKillip et al. 1989) as well as 
macro-pores within the resin (Gaefke et al. 2007), which diminishes the ultimate strength of the material. Increasing the temperature of the environment at which this reaction occurs, can benefit the PFA polymerization reaction by catalyzing it (i.e. accelerating the complete curing process of specimens, before the binder is evaporated from the specimen) and avoiding the presence of an undesirable amount of water inside the rock analogue pores, thereby producing stronger analogues.

The high-temperature curing process initiates during 3D printing. After each pass of the print head over the build platform, with the subsequent sand recoat, a heat emitter, at an average temperature of $40^{\circ} \mathrm{C}$, heats up each layer of the to-be-printed analogues. Furthermore, after the printing job has concluded, the specimens are placed inside a furnace at a temperature of $80^{\circ} \mathrm{C}$ for $24 \mathrm{~h}$. This specific temperature is chosen after analyzing the results of unconfined compressive strength (UCS) tests performed on specimens with no thermal treatment applied (i.e. curing at atmosphere conditions) and specimens that are treated with temperatures of up to $200{ }^{\circ} \mathrm{C}$. The specimens cured at $80{ }^{\circ} \mathrm{C}$ show the highest peak uniaxial strength and exhibit structure degeneration after $125 \mathrm{~h}$ of treatment, which is the longest duration among the other groups of specimens.

\subsection{Experimental Set Up}

The consolidated triaxial tests analyzed in this study were performed in the GeoREF facilities, at the University of Alberta. The hydrostatic confining stress was applied by pressurizing Xiameter ${ }^{\circledR}$ PMX-200 Silicone Fluid 100CS, which was also used as a pore fluid. The planned confining pressure for each test was achieved in a single loading process by steps of $2 \mathrm{MPa}$. Each compaction stage lasted until there was no appreciable variation on the readings of the axial and radial displacement measurement systems. At that point, before finishing the current stage, the specimens' permeability was calculated by measuring the resulting $\Delta \mathrm{P}$ from a constant flow rate.

An ENERPAC L1608 Hydraulic Cylinder mounted on a steel frame composes the loading system. Three TELEDYNE ISCO SFX 220 pumps apply pore (top-bottom) pressures and Cell Pressure (i.e. Confining Stress). During Permeability tests, an Ametek Chandler QUIZIX QX Pump is used for accurately pumping the pore fluid through the body of the specimen, while the Pore ISCO pump is designed as a receiver. The exact configuration of both the Frame and the Pumps is shown in Fig. 1. Two types of linear variable displacement transducer (LVDT) systems are set up for each specimen: two axial LVDTs for calculating axial displacement mounted on the top and bottom end caps of the specimen, with a range of $12 \mathrm{~mm}$ and an accuracy of $\pm 25.4 \mu \mathrm{m}$, and one radial LVDT for calculating radial displacement with a range of $6 \mathrm{~mm}$ and an accuracy of $\pm 25.4 \mu \mathrm{m}$. As support system, an external LP, with a range of $100 \mathrm{~mm}$ and repeatability of $\pm 2 \mu \mathrm{m}$, is attached to the RAM and mounted on top of the cell cap. Then, this entire configuration is enclosed in a NMC 6500 triaxial pressure cell with a maximum operating pressure of $44.8 \mathrm{MPa}(6500 \mathrm{psi})$.

To ensure complete saturation of the specimens, they were submerged for a week in a sealed container full of PMX-200 Silicone Fluid. Also, before setting up the triaxial test, the analogues were placed into a vacuum chamber at an average pressure of $-15 \mathrm{Psi}(-0.1 \mathrm{MPa})$, so the high suction pressure created around the specimen helped driving the oil into the remaining empty pores and contributed to completion of the imbibition process. Finally, after each specimen was placed inside the triaxial pressure cell, it was left for $24 \mathrm{~h}$ under an effective confining stress of $1 \mathrm{MPa}$ (resulting from a pore pressure of $2 \mathrm{MPa}$ and a confining pressure of $3 \mathrm{MPa}$ ) for pore media saturation purposes. 


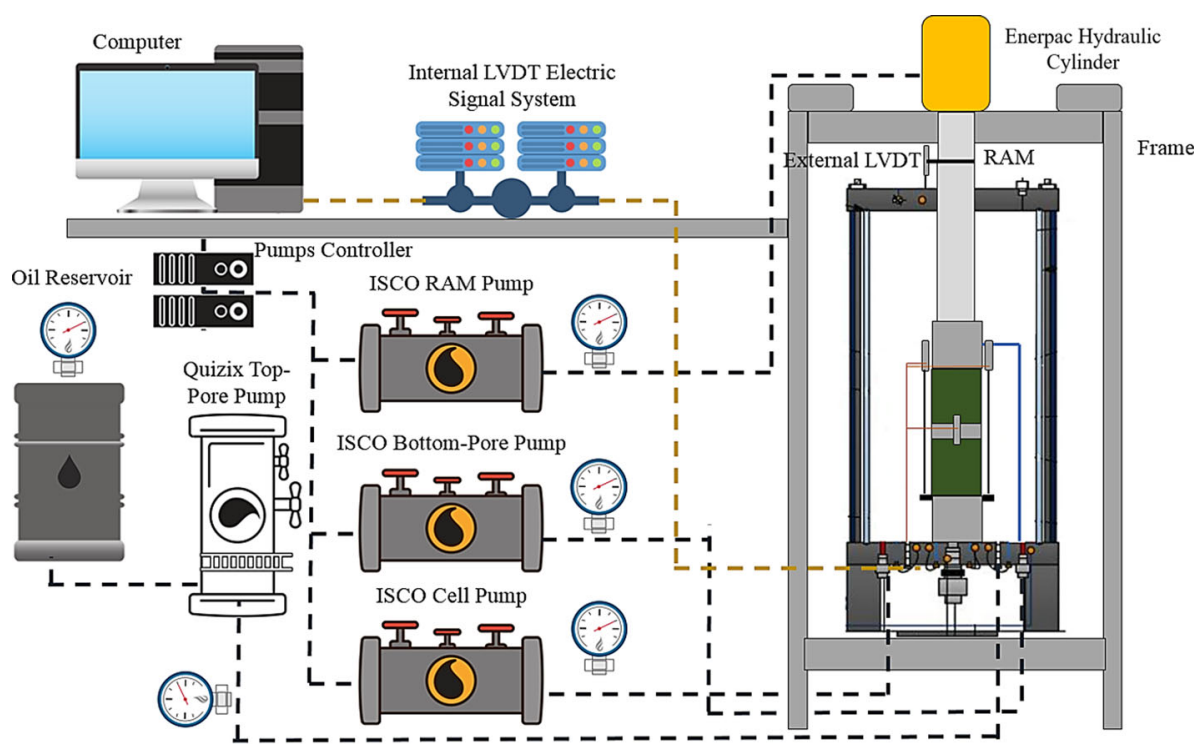

Fig. 1 Experimental set up used for performing the consolidated drained triaxial tests

\section{Results and Analysis of Mechanical Behaviour}

\subsection{Bulk Compressibility}

In this section, the numerical results from the hydrostatic compression stages of four consolidated drained triaxial tests were analyzed to extract information related to the bulk compressibility of the 3D printed sandstone analogues. The specimens were built using a $20 \%$ PFA saturation ( $4 \%$ of the bulk volume), $90^{\circ}$ layer orientation (layers perpendicular to load application) and a layer thickness of $250 \mu \mathrm{m}$. The maximum effective confining stress levels used in the tests were $10 \mathrm{MPa}, 18 \mathrm{MPa}, 25 \mathrm{MPa}$ and $30 \mathrm{MPa}$. The volumetric strain was calculated using the results obtained from the axial $\left(\varepsilon_{\mathrm{a}}\right)$ and radial $\left(\varepsilon_{\mathrm{r}}\right)$ deformations undergone by the specimens (Eq. 1).

$$
\varepsilon_{v}=\varepsilon_{\mathrm{a}}+2 \varepsilon_{\mathrm{r}}
$$

The sandstone analogues' bulk compressibility $\left(C_{b c}\right)$ are calculated using the relationship proposed by Zimmerman (1991), Eq. (2);

$$
C_{b c}=\frac{1}{V_{b}^{i}}\left(\frac{\partial V_{b}}{\partial P_{c}}\right)_{P_{\mathrm{p}}}
$$

where $V_{b}^{i}$ corresponds to the initial bulk volume of the specimen, calculated basically by determining the volume of the cylindrical analogue (i.e. $V_{b}^{i}=\pi r_{s}^{2} h_{s}$ ), and $\partial V_{b}$ is the change in bulk volume generated by an increase/decrease in the confining stress $(\partial P c)$ under a constant value of pore pressure, $P_{\mathrm{p}}$. To facilitate the analysis of this property, specimen's bulk compressibility is plotted against effective confining stress (Fig. 2).

The 3D printed sandstone analogues behave in the typical manner stated in the consolidation theory proposed by Terzaghi (1964). The non-linear reduction in the bulk compressibility of the specimens is the primary result of increasing bond breakage at the grain boundaries 


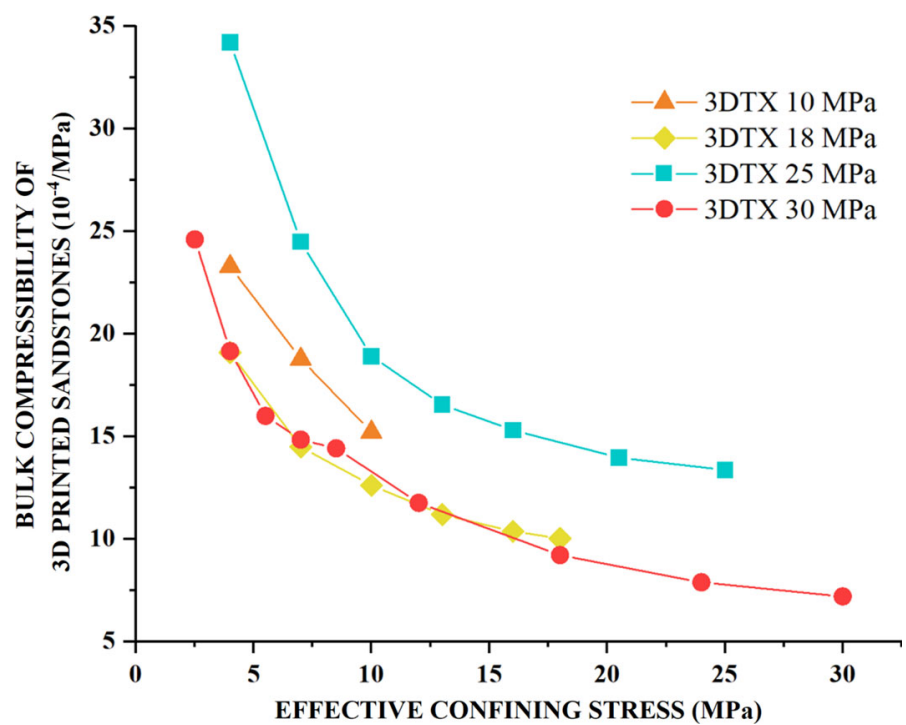

Fig. 2 Bulk compressibility curves for 3D printed sandstone analogues. The curve shows the behavior for the specimens subjected to stress levels of $10 \mathrm{MPa}, 18 \mathrm{MPa}, 25 \mathrm{MPa}$ and $30 \mathrm{MPa}$

due to increasing effective confining stress, which at the same time leads to a continuous porosity reduction. The rate of change of $C_{b c}$ with effective confining stress is very similar for all specimens, indicating that the specimens are showing a reasonable degree of repeatable compressibility (Fig. 2). The offset of the curves, in particular the test conducted at $25 \mathrm{MPa}$, is due to measurement errors in the initial volume of the specimen.

Experimental data gathered by Zimmerman (1992) from widely studied reservoirs such as Berea and Bandera sandstones is compared with the volumetric behavior of the $3 \mathrm{D}$ printed specimens (Fig. 3). Due to similarities on the range of confining stresses used during the tests, the data resulting from the sandstone analogue subjected to a maximum confining stress of $30 \mathrm{MPa}$ is used for building this plot. The values of this property for the $3 \mathrm{D}$ printed rocks lie within the same order of magnitude of the natural reservoir materials $\left(10^{-4} \mathrm{MPa}\right)$. This allows researchers to be optimistic about extrapolating the volumetric behavior of this synthetic material to the actual mechanical response of a natural reservoir rocks. However, the $3 \mathrm{D}$ printed sandstones are far more compressible than natural materials like Berea and Bandera sandstones, so there is a necessity to study additional methods to produce properties of the manufactured analogues closer to those of natural sandstones. The bulk compressibility of 3D printed specimens is around 6-7 times lower than the compressibility of a uniform natural material such as Berea Sandstone. This aspect can be directly related to the fact that $3 \mathrm{D}$ printed analogues have a porosity that is nearly equivalent to that of an unconsolidated sand ( 36\%), while typical reservoir rocks have porosities that range between 9 and $24 \%$. Therefore, $3 \mathrm{D}$ printed analogues are $50 \%$ more porous than most of the natural.

\subsection{Bulk Modulus and Matrix Deformation}

For any drained hydrostatic compression test, it is possible to determine the material's jacketed drained bulk (i.e. secant compression) modulus, $K$. For the case of the $3 \mathrm{D}$ printed sandstone 


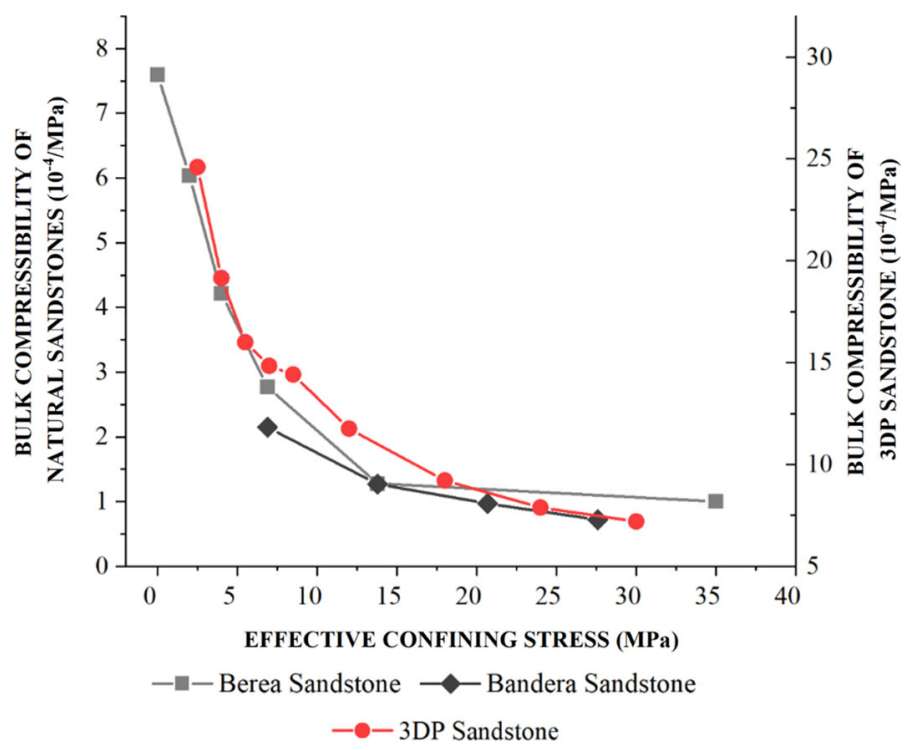

Fig. 3 Bulk compressibility curves from natural sandstones and 3D printed Sandstone analogues. The plot shows good agreement in the order of magnitude of bulk compressibility. Sandstone analogues are 6-7 times more compressible than Berea/Bandera sandstones

analogues, this property can be calculated by estimating the induced specimen's volumetric strain $\left(\Delta V / V_{o}\right)$ as a response to a correspondent increase in effective confining stress $(\Delta \sigma)$ (Eq. 3). The term "jacketed" comes from the fact that the specimen's porous media remains isolated from the confining fluid by a membrane or "jacket"

$$
K=\left.V_{o} \frac{\Delta \sigma}{\Delta V}\right|_{\text {jacketed }}
$$

where $V_{o}$ corresponds to the initial bulk volume of the specimen; $\Delta V$ the s the change in bulk volume generated by an increase/decrease as a response to a correspondent increase in effective confining stress $(\Delta \sigma)$.

The behavior of the 3D printed specimens correlates with the observations made by Ardila (2018), where, rock analogues are 3D printed using the same materials (i.e. silica sand and PFA). Specimens in this study experience a plastic irreversible matrix deformation under stress levels higher than 7.5 MPa (Fig. 4). This phenomenon is associated with the stressdependency of the compression modulus, attributed mainly to the closing of pre-existing micro-cracks in natural materials or weakly bonded zones in the $3 \mathrm{D}$ printed analogues. Variations in the value of $K$ for different specimens are attributed to variation in the volume of poorly-bonded zones in each analog (Hu et al. 2014). The lower the volume of these zones, the 'harder' the specimen, therefore, the higher its bulk modulus.

Fabre and Gustkiewicz (1997) state that the common range of bulk modulus for natural sandstones is $15-45 \mathrm{GPa}$, which are considerably higher values than the ones obtained for the $3 \mathrm{D}$ printed analogues (around $10 \%$ of the value for natural reservoir rocks), $0.3-1.5 \mathrm{GPa}$. The values for bulk moduli for different natural rocks such as marble, sandstone or limestone, also seem to correlate with mineralogical composition and rock porosity: the higher the porosity of the specimen, the lower its bulk modulus. 


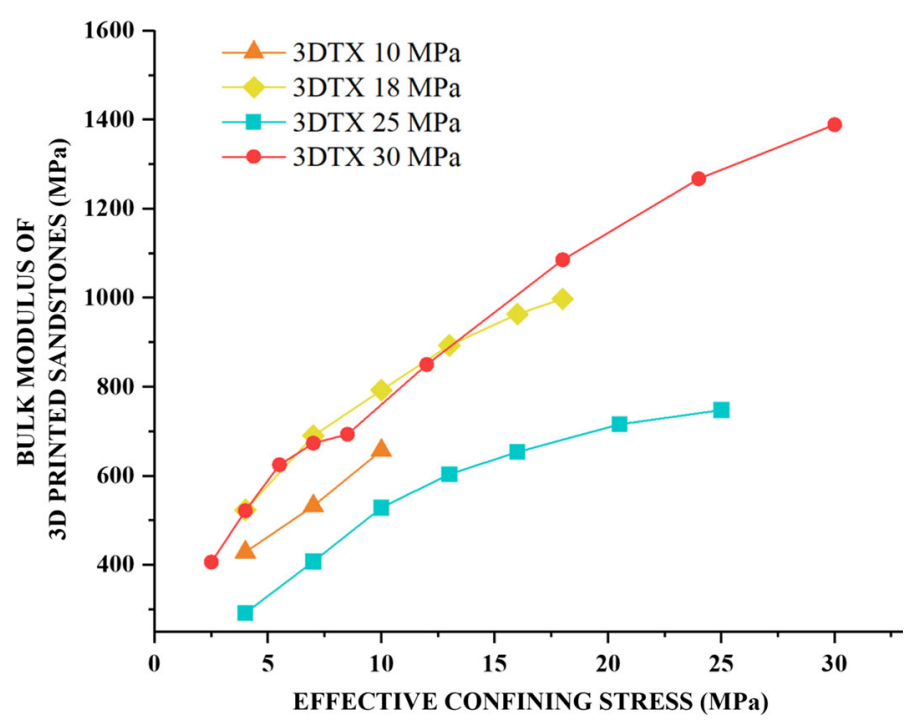

Fig. 4 Bulk modulus $(K)$ versus effective confining stress for 3D printed Sandstone Analogues. The plastic deformation regime of the $3 \mathrm{D}$ printed specimens is suspected to start in the vicinities of a confining stress of $5 \mathrm{MPa}$

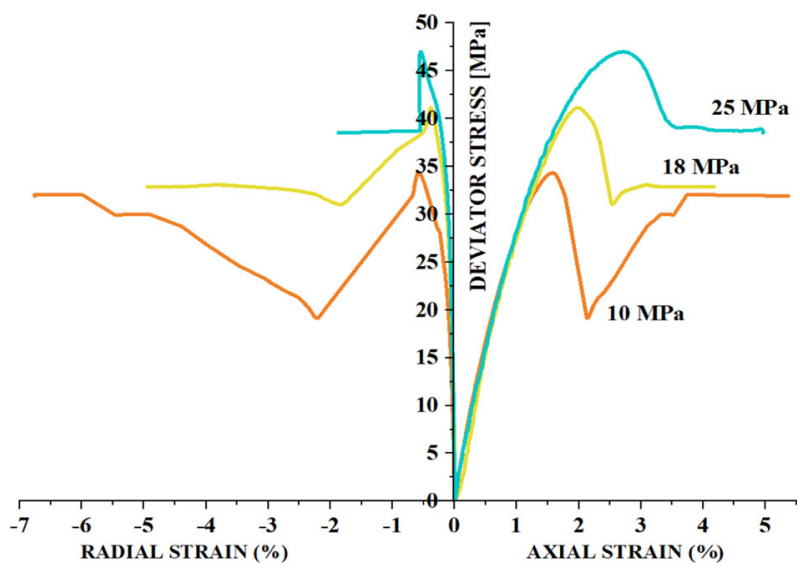

Fig. 5 Deviator stress versus strain for 3D printed specimens under different confining stresses. The specimens failed at 10 and $18 \mathrm{MPa}$ confining stress exhibited frictional strengthening in post-peak region. The specimen failed at $25 \mathrm{MPa}$ showed an elastic-perfectly plastic type of behavior

\subsection{Peak Strength and Stress-Strain Behavior}

Sections 3.3 and 3.4 did not contain the results from the specimen failed at $30 \mathrm{MPa}$ effective confining stress due to differences in its hydrostatic loading process with respect to the rest of the specimens. There is an equivalent behavior of the initial linear-elastic portion of the deviator stress against axial and radial strain for the three specimens. The deviator stress against axial and radial strain relationships for each triaxial tests are plotted in Fig. 5

The test results confirmed a direct relationship between the increase in confining stress and the peak deviator stress. The specimen subjected to $10 \mathrm{MPa}$ effective confining stress 
Table 1 Summary of results from CD triaxial testing on 3D printed sandstone analogues

\begin{tabular}{llllll}
\hline $\begin{array}{l}\text { Effective } \\
\text { confining stress } \\
(\mathrm{MPa})\end{array}$ & $\begin{array}{l}\text { Peak deviator } \\
\text { stress (MPa) }\end{array}$ & $\begin{array}{l}\text { Axial strain at } \\
\text { failure }(\%)\end{array}$ & $\begin{array}{l}\text { Radial strain at } \\
\text { failure }(\%)\end{array}$ & $\begin{array}{l}\text { Young's } \\
\text { Modulus (GPa) }\end{array}$ & Poisson's ratio \\
\hline 10 & 34.3 & 1.57 & 0.61 & 2.74 & 0.19 \\
18 & 41.1 & 2.28 & 0.38 & 2.86 & 0.16 \\
25 & 47.0 & 2.69 & 0.54 & 2.99 & 0.10 \\
\hline
\end{tabular}

failed at 34.3 $\mathrm{MPa}$, while the one subjected to $18 \mathrm{MPa}$ effective confining stress failed at 41.1 MPa, which implied an increase of $19.8 \%$. The specimen subjected to $25 \mathrm{MPa}$ effective confining stress failed at $47 \mathrm{MPa}$, which implied an increase of $37 \%$ with respect to the lower case. The initial elastic portion of the curve was almost identical, showing negligible variation between the stiffness of the specimens (Table 1).

The axial strain at peak conditions for each one of the CD Triaxial tests ranged between 1.5 and $2.7 \%$. The higher the confining stress at failure conditions, the higher the axial strain undergone by the specimens during the shearing process. Moreover, the behavior of the specimens on the post-peak region correlated with the observations made in other studies (Martin 1997; Hajiabdolmajid and Kaiser 2003; Diederichs et al. 2007; Cai et al. 2009). At high levels of confining stress (above $5 \mathrm{MPa}$ ), the failure mode of strong rocks was dominated by the frictional component of the strength.

As the cohesion weakening frictional strengthening model by Hajiabdolmajid et al. (2003) states, the progressive damage inflicted to the rock specimen during a destructive compression test is first sustained by the cohesive forces, which come from the bonding between the grains. Failure starts from the grain boundaries and the loss of the mobilized cohesive component is appreciated at the first decreasing slope after the peak is reached. Nevertheless, when significant further damage is imposed to the specimen along the failure plane, the frictional component of the strength is activated. The movement of the particles, due to the generated frictional forces from the readjustment of disintegrated blocks and the grain interlocking phenomena contributes to the recovery of part of the material's strength.

These concepts are clearly evidenced in Fig. 5, especially for the specimens failed at 10 and $18 \mathrm{MPa}$, showing a substantial increase on the post-peak stress values after the initial weakening, generated by the shear failure; with a tendency towards the residual strength. For the specimen failed at $25 \mathrm{MPa}$, there is no such marked frictional strengthening and the frictional forces maintain the stress (i.e. elastic-perfectly plastic behavior) until it starts a decreasing pattern towards residual strength. Finally, there is no initial softened slope attributed to any crack closure phenomenon. This pattern is probably generated due to previous consolidation stages, where the specimens are compacted, and the pore structure is deforming and closing any pre-existing void or poorly bounded zones.

\subsection{Volumetric Deformation}

The specimens sheared at 10 and $18 \mathrm{MPa}$ effective confining stress exhibited a volumetric behavior comparable to that of medium to dense natural sandstone: at the initial stages of the deviatoric stress application, the 3D printed sandstone analogues experienced compression (i.e. decrease in bulk volume). After reaching the values of 1.8 and $2.4 \%$ axial strain 


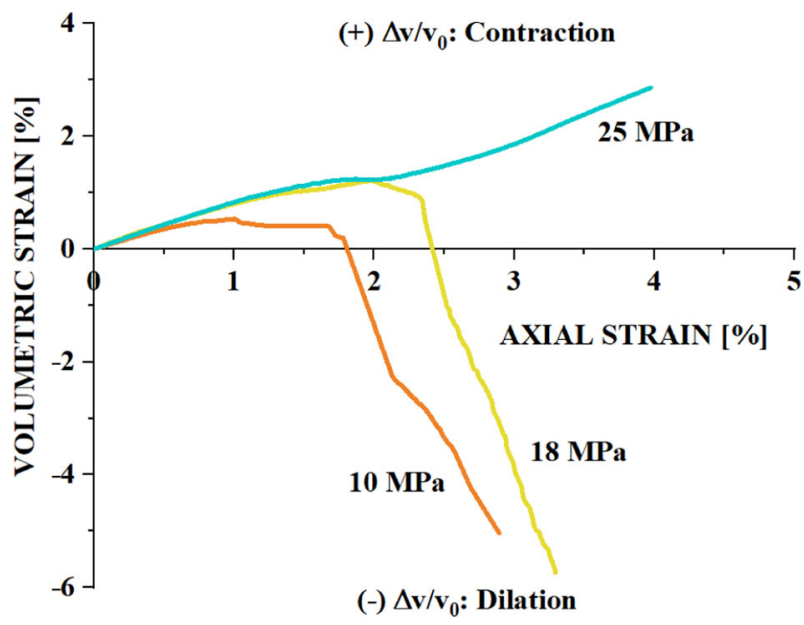

Fig. 6 Volumetric behavior of 3D printed analogues under triaxial conditions. The specimens failed at 10 and $18 \mathrm{MPa}$ showed a contractive behavior before failure and a persistent-dilation after reaching peak stress. The specimen failed at $25 \mathrm{MPa}$ showed a persistent-contractive behavior throughout the test

respectively, very close to failure conditions, they exhibited a remarkable increase in volume (dilation behavior), represented by a value of 5-6\% final volumetric strain after failure.

In contrast, the specimen sheared at $25 \mathrm{MPa}$ exhibits a purely contractive behavior. From the beginning of the test, the specimen undergoes a reduction in bulk volume during shearing. Unlike in natural medium-dense sandstones, this specimen does not display dilatant behavior after reaching failure conditions (Fig. 6).

\subsection{Failure Mode and Hoek and Brown Failure Criterion}

The triaxial cylinders failed at well-defined inclined shear planes at the angles between $55^{\circ}$ and $60^{\circ}$ (Fig. 7). Applying the linear Mohr-Coulomb theory, the inclination angle of the physical failure plane, $\beta$, was calculated based on the average inclination of the shear plane (Eq. 4). When combining the results obtained from the three triaxial tests, the result was an average friction angle, $\varphi$, of $29^{\circ}$, which theoretically corresponded to a typical value of a medium sand.

$$
\beta=45+\frac{\varphi}{2}
$$

The difference in color in Fig. 7 is attributable to the adjustments on the color saturation and brightness of the camera while taking the pictures. The real color of the specimens after completing the consolidated drained triaxial tests is most likely to be similar to the one exhibited by the specimen failed at $25 \mathrm{MPa}$ effective confining stress (Fig. 7).

Using the results of unconfined compressive strength tests, Direct Tension tests and consolidated drained triaxial tests performed on this sandstone analogues, it was possible to model the Hoek and Brown Failure criterion (Eq. 5) for the 3D printed sandstone analogues, using the Rocscience software, RocLab.

$$
\sigma_{1}^{\prime}=\sigma_{3}^{\prime}+\sigma_{c i}\left(m_{i} \frac{\sigma_{3}^{\prime}}{\sigma_{c i}}+s\right)^{a}
$$




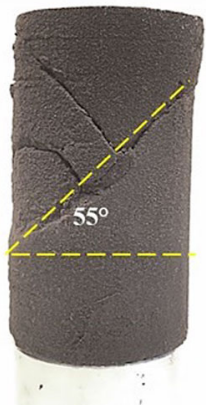

$10 \mathrm{MPa}$

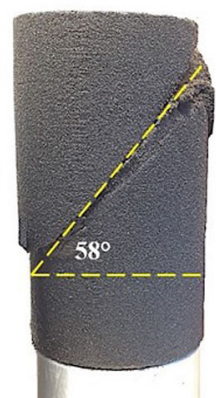

$18 \mathrm{MPa}$

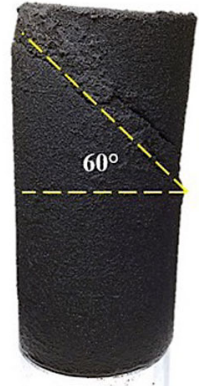

$25 \mathrm{MPa}$

Fig. 7 Failure modes of 3D printed specimens under different confining stress conditions

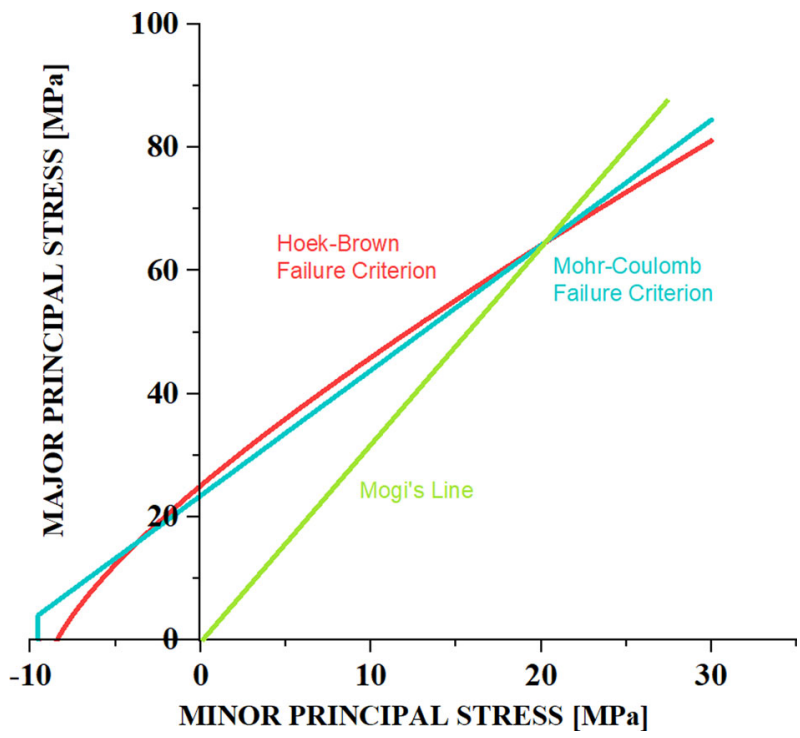

Fig. 8 Hoek and Brown failure criterion for 3D printed reservoir sandstones. This model indicates that 3D printed sandstone analogues exhibit a ductile-to-brittle failure mechanism's limit at around 17.5 MPa

where $\sigma_{1}^{\prime}$ and ' $\sigma_{3}$ are the maximum and minimum effective stresses failure respectively, $m_{i}$ is the values of the Hoek-Brown constant $m$ for the rock mass, $s$ and a are constants which depend upon characteristics of the rock mass, and $\sigma_{c i}$ is the uniaxial compressive strength of the intact rock.

To fulfill the requirements of the calculation software, the Geological Strength Index (GSI) for the sandstones was established as 100 (i.e. the value for intact material without discontinuities or fractures). The calculated intact uniaxial strength was $25.7 \mathrm{MPa}$ and the $m_{i}$ was 2.63, which was slightly lower than the results obtained for natural sandstones ( 13-21, according to Hoek 2006). The blue line showed the corresponding Mohr-Coulomb failure criterion for this material, while the red line was the Hoek-Brown failure criterion (Fig. 8). The green line corresponded to the Mogi's line, defined by Mogi in 1966. This line indicated the limit at which the failure mechanism of the rock material changed from brittle dominated 
(to the left) to ductile dominated (to the right). For 3D printed sandstone analogues, this phenomenon occurred around $\sigma_{3}^{\prime}=17.5 \mathrm{MPa}$. These results were in good agreement to what was observed in the consolidated drained triaxial tests, where the effects of the frictional strengthening at $18 \mathrm{MPa}$ confining stress were reduced until reaching an almost perfectlyplastic post-peak residual behavior at $25 \mathrm{MPa}$.

The specimens failed under 10 and $18 \mathrm{MPa}$ confining stress exhibited a frictional strengthening behavior after reaching peak stress. Although this was not a typical brittle behavior, it might be a result of the high porosity of the material, which favored grain re-organization in response to stress. The failure process due to shear localization, expressed in well-defined fracture planes, might validate the results obtained from the Hoek-Brown model. On the other hand, the specimen failed at $25 \mathrm{MPa}$ confining stress (within the ductile failure regime of the Hoek-Brown model), also failed by shear localization. However, the persistent compaction behavior of its volumetric strain curve was indeed typical of a ductile failure.

The failure behavior of the $25 \mathrm{MPa}$ specimen may be attributed to a complex mixedfailure behavior resultant from the progressive brittle rupture of the PFA necks between the grains and the continuous friction developed between the silica sands grains due to stressinduced movement and re-accommodation on surrounding pores. Low confining stresses allow considerable grain movement and frictional inter-locking while high confining pressure environments hinders them. In both cases, after fracture coalescing process is critical, the failure planes are aggressively developed along the weakened brittle PFA bounding necks, which favors the formation of clear shear planes even at high confining pressures.

\section{Hydraulic Behavior Results}

At the end of each consolidation stage during the hydrostatic compression procedure of the 3D printed specimens, a series of permeability tests were performed to study the evolution of the hydraulic properties of the four sandstone analogues with the correspondent variation of the confining stress conditions. These tests were performed at a constant flow rate delivered by an Ametek Chandler QUIZIX QX Pump and the differential pressure created across the body of the specimen was recorded using a differential pressure transducer (Validyne DP15TL) with maximum capacity of $550 \mathrm{kPa}$ and accuracy of $\pm 2.75 \mathrm{kPa}$. After reaching stable flow rates, the total time and quantity of fluid discharge and differential pressure were measured and recorded to calculate the permeability.

The permeability of the 3D printed specimens reduces with the increase of the effective confining stress (Fig. 9). This behavior has been well documented since the canonic study by David et al. (1994). Additionally, the reduction in permeability seems to be more accentuated at lower stress levels, which corresponds to the behavior observed in natural materials because of the crack closure phenomenon (David et al. 2001). As previously pointed for the compressibility study, this behavior can be attributed to the compaction of weakly-bound zones throughout the body of the 3D printed specimens.

The initial permeability of the 3D printed sandstone analogues range between 800 and $1000 \mathrm{mD}$; for higher effective confining stresses (20 MPa), its value lowers down to $200 \mathrm{mD}$. Natural rocks such as Berea sandstone have a permeability that ranges between 114 and $1.168 \mathrm{mD}$, with porosities that vary between 19 and 26\% (Churcher et al. 1991). Fontainebleau sandstones have a permeability that ranges between 12.5 and $704 \mathrm{mD}$, with porosities that fluctuate between 7 and 17\% (Gomez et al. 2010). 3D printed sandstone analogues have permeability values that are comparable in scale with the results obtained from well-studied 


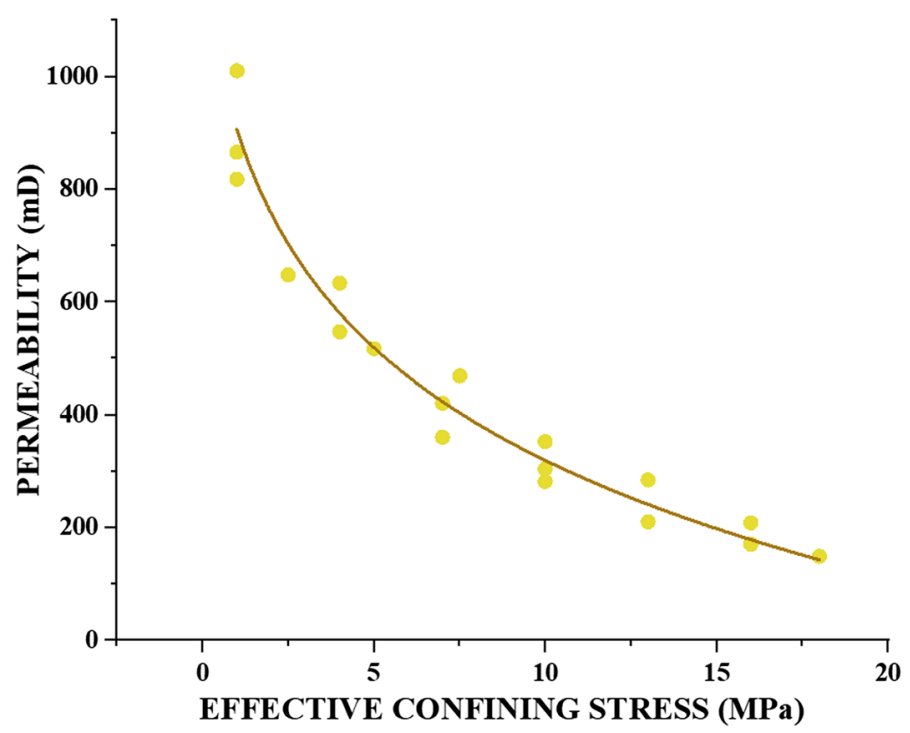

Fig. 9 Evolution of the permeability of the 3DP sandstone analogues with confining stress. Yellow bullet points correspond to the actual permeability measurements and solid line to the exponential curve fitting

natural reservoir rocks. Additionally, rock analogues manufactured with the printing properties presented in this study resemble the properties of highly-porous natural sandstones.

As it was proposed by Pedrosa (1986), the sensitivity of the pore structure of natural reservoir sandstones under hydrostatic compaction processes, i.e. permeability evolution with effective pressure; can be successfully described by the pressure sensitivity parameter, $\gamma$ (Eq. 6). By using the empirical law exposed by this expression, the permeability data extracted from the consolidated drained triaxial tests can be related to the incremental effective pressure and the parameter $\gamma$ can be calculated by using an exponential fitting. The correspondent permeability reduction is then plotted against effective confining stress (Fig. 10).

$$
k=k_{o} \exp \left(-\gamma \sigma^{\prime}\right)
$$

where $k$ is the permeability of a porous material at any stress condition, $k_{o}$ is the permeability of the material at an undisturbed state and $\sigma^{\prime}$ is any specific effective stress condition at which the permeability is calculated.

A compilation of experimental results published by David et al. (1994), shows that the pressure sensitivity parameter, $\gamma$ ranges from 0.001 and $0.020 \mathrm{MPa}^{-1}$ for most granular materials; and from 0.023 to $0.11 \mathrm{MPa}^{-1}$ - for low porosity rocks such as tight sandstones or fractured rocks. David et al. (2001) also present the correlation between high $\gamma$ values and sharper reduction of permeability with increasing effective pressure. The results encountered for 3D printed sandstone analogues are between 0.103 and $0.118 \mathrm{MPa}^{-1}$, which corresponds to highly sensible materials.

While the failure mode of the 3D printed specimens was located in the brittle field, i.e. shear localization materialized in a failure plane, the high-pressure sensitivity of the specimens might be explained by a heterogeneous damage concentration, preferentially in weakly bound zones. During hydrostatic compaction, the brittle failure behavior of the cured Furan resin (Gaefke et al. 2007) could induce continuous rupture of bounding necks, which reduced the structural stability of specific regions of the specimens. This phenomenon translated into 


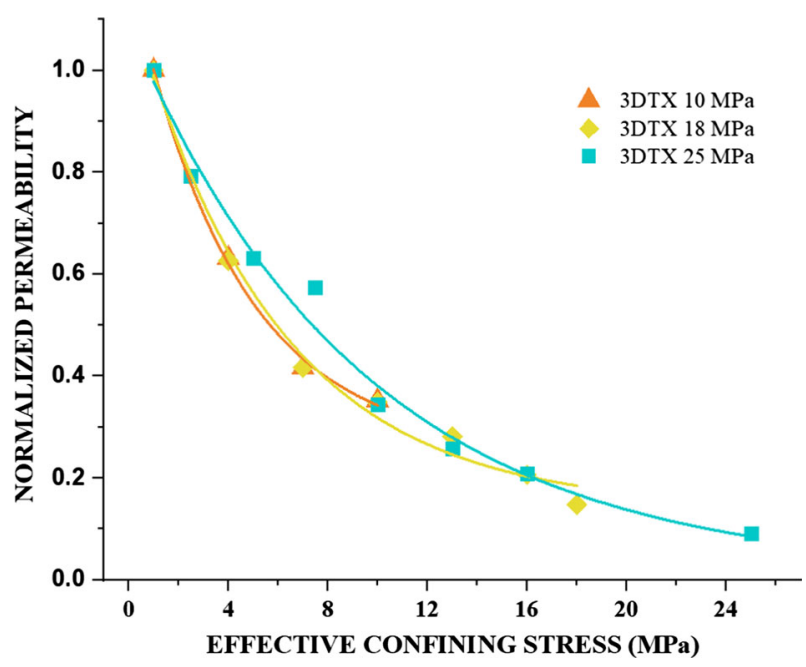

Fig. 10 Permeability reduction ratio for 3D printed Specimens. The curve-fitting of the experimental data from the 3D printed specimens with the relationship proposed by Pedrosa et al. (1986), indicate that sandstone analogues are a highly pressure-sensitive material

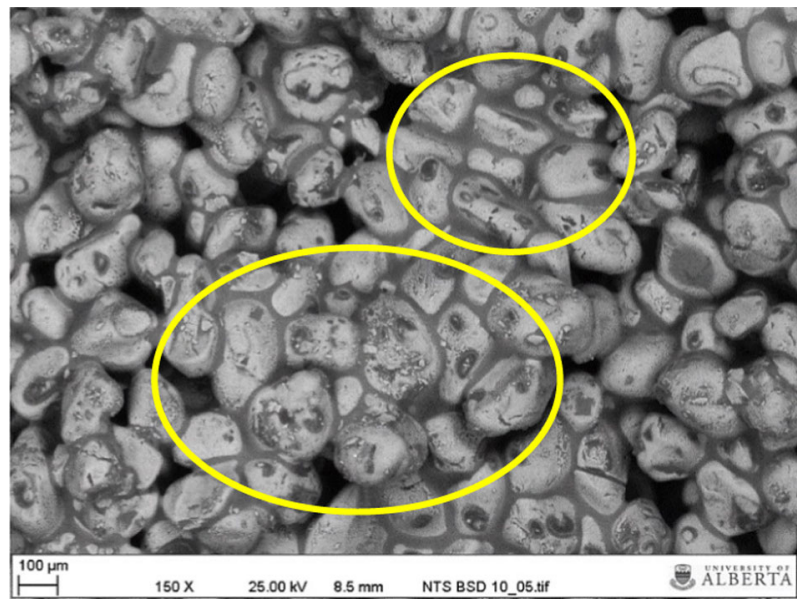

Fig. 11 SEM Imaging of Deformed 3D printed Specimen's Pore structure. The sections circled in yellow represent zones with a noticeable grain agglutination

progressive grain re-accommodation and accretion and therefore permeability reduction. In addition, at a high-confining pressure $(25 \mathrm{MPa})$, the volumetric behavior of the $3 \mathrm{D}$ printed sandstone analogues exhibited a progressive compaction, which could also be a trigger to the high permeability decrement observed in this analogue material.

SEM images with a resolution of $100 \mu \mathrm{m}$ were taken from 3D printed specimens subjected to an effective confining pressure of $10 \mathrm{MPa}$ (Fig. 11). The PFA bounding necks between grains were depicted as the darker-gray zones, while the silica sand grains were of a lighter gray color. The sections circled in yellow showed zones with a noticeable grain agglutination and the black zones represent porous space. The phenomenon observed in this 
image indicated the high compressibility and the correspondent permeability reduction of the 3D printed specimens. Stress-induced grain agglutinations resulting from progressive pore collapse implied remarkable volumetric strains and flow path closure within the specimens' porous media.

\section{Conclusions}

The compressibility of the $3 \mathrm{D}$ printed sandstone analogues has the same order of magnitude as a natural reservoir sandstone. Nevertheless, due to the high porosity of the 3D printed silica sand $(\sim 35.7 \%)$, volumetric deformation of rock analogues is around 6 times higher than in Berea Sandstone. Additionally, during the isotropic compression stages the onset of crack closure is observed at above $7.5 \mathrm{MPa}$ effective confining stress. This evidence is evident in a change in the slope of the Bulk modulus curve.

Consolidated drained triaxial tests conducted on the 3D printed sandstone analogues revealed that their peak shear stress had a direct relationship with the effective confining stress conditions at failure: the higher the effective confining stress at failure, the higher the strength of the specimen. This trend was similar to the results encountered in natural reservoir sandstones. The failure mode of the specimens was supported by a defined shear plane inclined at around $60^{\circ}$, which corresponded to a friction angle of $30^{\circ}$. The specimens sheared at $10 \mathrm{MPa}$ and $18 \mathrm{MPa}$ confining stresses exhibited at a volumetric behavior comparable to a medium to dense sand, while the specimen sheared at $25 \mathrm{MPa}$ showed a persistent compaction volumetric behavior. The failure behavior of the 3D printed sandstone analogues followed the cohesion weakening friction strengthening model (Hajiabdolmajid et al. 2003).

The 3D printed specimens have proven to be highly sensible materials for studying the variation of effective confining pressure. The parameter $\gamma$, used to characterize this property was calculated to be within $0.103-0.118 \mathrm{MPa}^{-1}$. This phenomenon was explained by the progressive brittle failure of the Furan bounding necks, which generated concentrated damage across the specimens and consequently permeability reduction.

The results obtained from this study will reinforce the recommended path for future work on mechanical properties of 3D printed rock analogues. Researchers can expect more competent specimens when increasing the binder saturation used to build the $3 \mathrm{D}$ printed specimens. Future research work shall be focused mainly on testing suitable methods to decrease the interstitial porosity of the specimens. This might help 3D printing more natural rock-like porous media and achieve a more realistic order of magnitude on their mechanical behavior. Moreover, there is a necessity to understand the pore compressibility and mechanical behavior of this type of material at low effective confining stresses (i.e. 1-5 MPa). These fundamental avenues help establishing a comprehensive protocol to define the standards on mechanical testing of 3D printed materials and define the sensitivity of 3D printed analogues to each printing feature, avoiding anisotropy in the testing results.

Certainly, 3D printing technology using silica sand can be a feasible solution to replicate rock analogues, which are suitable for performing experimental investigation. The results obtained in this study strongly suggest that even though $3 \mathrm{D}$ printed specimens are more compressible and permeable than natural sandstones, this technology might be suitable to provide an answer to engineering problems in reservoir geomechanics related to the repeatability of the experimental results, specimen-to-specimen homogeneity, scale-up issues, and pore-structure variability. 
Acknowledgements The authors gratefully acknowledge the financial sponsorship of the Energi Simulation Industrial Research Consortia in Reservoir Geomechanics for Unconventional Resources and the Reservoir Geomechanics Research Group [RG ${ }^{2}$ at the University of Alberta for their invaluable technical support.

Open Access This article is distributed under the terms of the Creative Commons Attribution 4.0 International License (http://creativecommons.org/licenses/by/4.0/), which permits unrestricted use, distribution, and reproduction in any medium, provided you give appropriate credit to the original author(s) and the source, provide a link to the Creative Commons license, and indicate if changes were made.

\section{References}

Ardila, N.A.: Hydraulic properties characterization of 3D printed sandstone analogues, Edmonton. (2018)

Bewick, R.P., Kaiser, P.K.: Influence of rock mass anisotropy on tunnel stability. In: Rockeng09, pp. 1-12. (2009)

Cai, M.: Practical estimates of tensile strength and Hoek-Brown strength parameter mi of Brittle Rocks. Rock Mech. Rock Eng. 43, 167-184 (2009). https://doi.org/10.1007/s00603-009-0053-1

Churcher, P.L., French, P.R., Shaw, J.C., Schramm, L.L.: Rock properties of berea sandstone, baker dolomite, and indiana limestone. SPE Int. Symp. Oilf. Chem. (1991). https://doi.org/10.2118/21044-ms

David, C., Menendez, B., Zhu, W., Wong, T.F.: Mechanical compaction, microstructures and permeability evolution in sandstones. Phys. Chem. Earth, Part A Solid Earth Geodesy 26, 45-51 (2001). https://doi.o $\mathrm{rg} / 10.1016 / \mathrm{s} 1464-1895(01) 00021-7$

David, C., Wong, T.F., Zhu, W., Zhang, J.: Laboratory measurement of compaction-induced permeability change in porous rocks: Implications for the generation and maintenance of pore pressure excess in the crust. Pure Appl. Geophys. 143, 425-456 (1994)

Diederichs, M.S.: The 2003 Canadian Geotechnical Colloquium : mechanistic interpretation and practical application of damage and spalling prediction criteria for deep tunnelling, pp. 1082-1116 (2007). https:// doi.org/10.1139/t07-033

Ex-ONE: Ex-ONE M-Flex ${ }^{\mathrm{TM}}$ User Manual version 1.02.07, (2014)

Fabre, D., Gustkiewicz, J.: Poroelastic properties of limestones and sandstones under hydrostatic conditions. Int. J. Rock Mech. Min. Sci. Geomech. Abstr. 34, 127-134 (1997). https://doi.org/10.1016/s0148-9062 (96)00037-X

Gaefke, C.B., Botelho, E.C., Ferreira, N.G., Rezende, M.C.: Effect of furfuryl alcohol addition on the cure of furfuryl alcohol resin used in the glassy carbon manufacture. J. Appl. Polym. Sci. 106, 2274-2281 (2007). https://doi.org/10.1002/app

Gomez, C.T., Dvorkin, J., Vanorio, T.: Laboratory measurements of porosity, permeability, resistivity, and velocity on Fontainebleau sandstones. Geophysics 75, E191 (2010). https://doi.org/10.1190/1.3493633

Gomez, J.S., Ardila, N., Chalaturnyk, R.J., Zambrano-Narvaez, G.: Reservoir geomechanical properties characterization of 3D printed sandstone. In: Proceedings of the VI Biot's Conference in Poromechanics, pp. 952-960. ASCE, Paris (2017)

Hajiabdolmajid, V., Kaiser, P., and Martin, C.D.: Mobilised strength components in brittle failure of rock. Geotechnique. 53, 327-336 (2003)

Hajiabdolmajid, V., Kaiser, P.: Brittleness of rock and stability assessment in hard rock tunneling. Tunn. Undergr. Space Technol. 18, 35-48 (2003)

Head, D., Vanorio, T.: Effects of changes in rock microstructures on permeability: 3-D printing investigation. Geophys. Res. Lett. (2016). https://doi.org/10.1002/2016g1069334.received

Hoek, E.: Practical Rock Engineering. North Vancouver, British Columbia (2006)

Hu, D.W., Zhang, F., Shao, J.F.: Experimental study of poromechanical behavior of saturated claystone under triaxial compression. Acta Geotech. 9, 207-214 (2014). https://doi.org/10.1007/s11440-013-0259-y

Ishutov, S., Hasiuk, F.J., Harding, C., Gray, J.N.: 3D printing sandstone porosity models. Interpret. 3D Vis 3, SX49-SX61 (2015). https://doi.org/10.1190/int-2014-0266.1

Jiang, C., Zhao, G.-F.: A preliminary study of 3D printing on rock mechanics. Rock Mech. Rock Eng. 48, 1041-1050 (2015). https://doi.org/10.1007/s00603-014-0612-y

Lee, C.S., Kim, S.G., Kim, H.J., Ahn, S.H.: Measurement of anisotropic compressive strength of rapid prototyping parts. Mater. Process. Technol. 187-188, 627-630 (2007). https://doi.org/10.1016/j.jmatprotec.2 006.11 .095

Marinos, P., Hoek, E.: Estimating the geotechnical properties of heterogeneous rock masses such as flysch. Bull. Eng. Geol. Environ. 60, 85-92 (2001). https://doi.org/10.1007/s100640000090 
Martin, C.D.: Seventeenth Canadian geotechnical colloquium: the effect of cohesion loss and stress path on brittle rock strength. Can. Geotech. J. 34, 698-725 (1997)

McKillip, W.J.: Chemistry of Furan Polymers. Adhes. Renew. Resour. (1989). https://doi.org/10.1021/bk-19 89-0385.ch029

Menezes, F.F., Lempp, C.: On the structural anisotropy of physical and mechanical properties of a Bunter Sandstone. J. Struct. Geol. 114, 196-205 (2018). https://doi.org/10.1016/j.jsg.2018.06.010

Pedrosa, O.A.: Pressure transient response in stress-sensitive formations. Soc. Pet. Eng. AIME, SPE. 2, 203-214 (1986). https://doi.org/10.2118/15115-ms

Primkulov, B., Chalaturnyk, J., Chalaturnyk, R., Narvaez, G.Z.: 3D printed sandstone strength : curing of furfuryl alcohol resin-based sandstones. 3D Print. Addit. Manuf. 4-3: 149-155 (2017). https://doi.org/1 0.1089/3dp.2017.0032

Rankin, T.M., Giovinco, N.A., Cucher, D.J., Watts, G., Hurwitz, B., Armstrong, D.G.: Three-dimensional printing surgical instruments: are we there yet? J. Surg. Res. 189, 193-197 (2014). https://doi.org/10.10 16/j.jss.2014.02.020

Terzaghi, K.: Theoretical soil mechanics. Géotechnique 14, 1-13 (1964). https://doi.org/10.1680/geot.1964.1 4.1.1

Tymrak, B.M., Kreiger, M., Pearce, J.M.: Mechanical properties of components fabricated with open-source 3-D printers under realistic environmental conditions. Mater. Des. 58, 242-246 (2014). https://doi.org/1 0.1016/j.matdes.2014.02.038

Zimmerman, R.W.: Compressibility of Sandstones, Amsterdam (1991) 